\title{
Enseigner les langues dans des contextes plurilingues: réflexions socio-didactiques sur le français en Suisse italienne
}

Roberto Paternostro ${ }^{1, a}$

${ }^{1}$ Université de Genève - ELCF, Rue de Candolle 5, 1211 Genève 4, Suisse

\begin{abstract}
Résumé. Le français jouit d'un statut particulier dans le paysage sociolinguistique de la Suisse : il y est à la fois langue première, seconde et étrangère. Néanmoins, cette tripartition ne rend que partiellement compte de la complexité du contact linguistique et de son impact sur l'enseignement/apprentissage des langues. Ainsi, cet article questionnera la pertinence de ces catégorisations à partir d'une approche didactique intégrée et contextualisée. L'analyse de la place du français et de son enseignement en Suisse italienne et la présentation d'une enquête menée auprès de lycéens seront l'occasion de s'interroger quant à l'adoption de dispositifs didactiques stimulant la réflexion et sensibilisant à la variation, dans le but d'éveiller l'intérêt des élèves envers une langue qu'ils ont en partage et de promouvoir le plurilinguisme et la diversité culturelle.
\end{abstract}

\begin{abstract}
French language enjoys a particular status in the sociolinguistic landscape of Switzerland. Indeed, it can be considered at the same time as a first language, as a second and as a foreign one. Nevertheless, such a tight categorization does not allow either taking into account the complexity of language contact or considering its impact on language learning. Thus, in this paper, the relevance of these categories will be questioned on the basis of an integrated and contextualized didactic approach. Furthermore, the analysis of the place of French language and teaching in the Italian speaking region of Switzerland will be an opportunity to question the introduction of didactic tools which stimulate reflection and raise the awareness of variation in order to awake the learners' interest towards a shared language and promote multilingualism and cultural diversity.
\end{abstract}

\section{Introduction}

Le paysage sociolinguistique de la Suisse constitue un observatoire privilégié du français en situation de contact et représente un terrain fécond pour le développement d'approches didactiques adaptées aux situations plurilingues et multiculturelles (voir Lüdi \& Werlen [18]). En effet, le français est en Suisse à la fois langue première (FLM/FL1), pour la Suisse romande, langue seconde (FLS), pour les locuteurs bilingues, pour les migrants installés de longue date et pour ceux qui utilisent régulièrement le français dans leurs pratiques de socialisation et de travail, et langue étrangère (FLE) pour les locuteurs des autres langues nationales et pour les migrants nouvellement arrivés. Cette tripartition, cependant, ne rend compte que partiellement des situations de contact, qui ont pour effet de complexifier la notion de frontière, ce qui n'est pas sans conséquences pour l'enseignement du français.

Cet article, qui s'inscrit dans un projet plus vaste ${ }^{1}$, se donne pour objectif de questionner les limites d'une telle classification et de montrer l'avantage d'une approche didactique intégrée et contextualisée (voir plus loin), qui appréhende l'amplitude des français de Suisse (FLM/FL1, FLS, FLE) sur un continuum favorisant le décloisonnement et l'intégration de ces catégories. Cette réflexion théorique s'appuiera sur l'analyse de la place du français et de son enseignement en Suisse italienne, notamment à partir des aspects socio-institutionnels et d'une enquête menée auprès de lycéens, qui nous permettra de mieux cerner les attitudes et les représentations vis-à-vis du français et de ses variations et de nous interroger quant à leur impact sur l'apprentissage.

Notre article s'organise comme suit : après une présentation générale de la situation sociolinguistique du français en Suisse et de ses retombées didactiques, nous nous attacherons à décrire la place du français dans le système éducatif tessinois. Puis, à partir de la présentation et de la discussion de notre enquête auprès de lycéens à Locarno, nous essaierons de montrer l'intérêt d'adopter des dispositifs didactiques stimulant la réflexion et sensibilisant à la variation, dans le but d'éveiller l'intérêt des élèves envers une langue qu'ils ont en partage et de promouvoir le plurilinguisme et la diversité culturelle.

\section{Le statut du français en Suisse : quelles retombées pour l'enseignement ?}

Lorsqu'on s'attache à décrire la situation sociolinguistique de la Suisse, on se limite souvent à mentionner le principe de territorialité des langues inscrit dans la Constitution fédérale, qui confère à chaque canton la faculté de définir sa langue

\footnotetext{
${ }^{\text {a }}$ Roberto.Paternostro@unige.ch

${ }^{1}$ Le projet «Français en Suisse | Français de Suisse. Place, statut et enjeux socio-didactiques de l'enseignement du FLS / FLE en Suisse » est un projet post-doctoral soutenu financièrement par les fonds overhead du Fond National Suisse de la Recherche Scientifique et par la Faculté des Lettres de l'Université de Genève. Son objectif est de réfléchir à la présence, au statut et aux enjeux de l'enseignement du français langue seconde et étrangère dans le contexte helvétique. À partir d'enquêtes de terrain menées dans plusieurs cantons auprès des élèves-apprenants et des enseignants de français, il s'agit d'analyser des problématiques telles que la norme pédagogique à privilégier, la définition d'objectifs contextualisés, la mise au point de dispositifs adaptés.
} 
officielle et qui pose les fondements de l'existence de régions linguistiques juxtaposées (voir, entre autres : CordierGauthier \& Dion [6] : 34 ; Grin [14]). Or, non seulement cette territorialisation des langues ne suffit pas à expliquer la complexité de la situation helvétique, mais elle ne correspond pas toujours à la réalité vécue par les locuteurs dans leurs pratiques quotidiennes. En ce qui concerne le français ${ }^{2}$, sa spécificité en Suisse relève en effet moins de sa dimension politico-administrative que de son statut de langue « en partage » ${ }^{3}$, aux multiples facettes, en situation de contact avec d'autres langues, notamment l'allemand, l'italien et le romanche, mais aussi les langues d'héritage parlées par les populations issues de la migration. Ces facettes, qui représentent les diverses actualisations des français de Suisse, loin d'être des réalités étanches, gagneraient à être appréhendées sur un continuum allant du français langue première au français langue étrangère, en passant par le français langue seconde. Un exemple particulièrement significatif, qui illustre bien cette condition de partage, est sans doute le «français fédéral». Cette expression, souvent utilisée de manière péjorative pour indiquer le français fautif de l'administration bernoise, est aussi utilisée pour désigner le français parlé par les Suisses allemands, avec une influence audible des dialectes alémaniques (Thibaut [27]). Il s'agit d'une forme de français de moins en moins stigmatisée, dans tous les cas reconnue, qui fait désormais partie intégrante du paysage linguistique de la Suisse.

\subsection{Vers une intégrative et contextualisée de la didactique du français}

Ainsi conçue, l'amplitude des français de Suisse se caractérise comme un observatoire privilégié de la dynamique du contact des langues. Elle constitue aussi un terrain fécond pour le développement d'une vision intégrative et contextualisée de la didactique du français (Roulet [25] ; Dabène [7] ; De Pietro \& Rispail [10]) favorisant la prise en compte de la variabilité et de la diversité linguistiques.

Les approches intégrées, qui font plus globalement partie des approches plurielles, sont « des approches didactiques qui mettent en œuvre des activités d'enseignement-apprentissage qui impliquent à la fois plusieurs (= plus d'une) variétés linguistiques et culturelles» (Candelier et al. [4]: 6) ${ }^{4}$. Par "variétés linguistiques », ces auteurs entendent en principe des langues différentes. Nous reprenons cette définition tout en proposant de l'élargir aux différentes variétés d'une seule et même langue (voir, Dabène \& Rispail [8] ; Cadet \& Guerin [3]), et de la situer dans le cadre plus vaste d'une didactique contextualisée. Partant d'une analyse fine des contextes sociolinguistiques, culturels et éducatifs, cette approche cherche à élaborer des dispositifs didactiques (théoriques et appliqués) adaptés à des contextes complexes. Elle vise ainsi une prise en compte plus efficace des terrains socio-didactiques en s'interrogeant sur les représentations et les pratiques linguistiques et pédagogiques des acteurs concernés (apprenants, enseignants, etc.) et en rendant compte de la variation (régionale, sociale $)^{5}$ en tant que génératrice de la diversité des situations d'apprentissage.

Reconnaître la porosité des frontières à l'intérieur même du français pourrait, en effet, encourager un dialogue bénéfique et offrir des réponses plus adéquates aux exigences didactiques des contextes plurilingues et multiculturels. La prise en compte des diverses facettes du français en Suisse (FLM/FL1, FLS, FLE) et de ses différentes variations constituerait, en effet, un moyen de stimuler la réflexivité des apprenants-locuteurs et de les aider à avancer dans l'appréhension et dans la gestion de la diversité linguistique et culturelle (voir: De Pietro [9] ; Guerin [17] ; Paternostro, [21]). Enfin, la prise de conscience que le français n'est pas une langue « étrangère » à leur univers, motiverait les apprenants à aller au-delà des seuls aspects communicationnels tout en développant des attitudes positives face au français et à son apprentissage.

\subsection{Langue « nationale » vs. langue « étrangère " ?}

D'un point de vue institutionnel, un important travail a déjà été réalisé en Suisse, grâce notamment à la collaboration de linguistes et didacticiens. L'existence d'organismes intercantonaux, comme la Conférence Intercantonale de l'Instruction Publique de la Suisse romande et du Tessin (CIIP), qui réunit les Conseillères et Conseillers d'état en charge de l'éducation des cantons francophones (Genève, Jura, Neuchâtel, Vaud), italophone (Tessin) et bilingues

français-allemand (Berne, Fribourg, Valais), est une illustration du statut de « langue en partage » que le français revêt dans le pays. Cependant, ce statut se rencontre de façon moins explicite dans certains textes officiels qui ne relèvent pas directement de la sphère éducative. Ces textes, en effet, proposent une classification des langues du pays en trois catégories: les langues nationales (définies ainsi par la Constitution fédérale ${ }^{6}$ ), les langues de scolarisation (qui

\footnotetext{
${ }^{2}$ Nous n'aborderons pas la question des autres langues nationales, d'une part, parce que c'est le français qui nous intéresse ici et, d'autre part, parce que l'allemand, et dans une moindre mesure l'italien, connaissent des situations de diglossie, qui complexifient le paysage (Christen [5] ; Pandolfi [20]).

3 À l'instar d'institutions francophones telles que l'Organisation Internationale de la Francophonie (www.francophonie.org), nous utilisons cette expression dans le sens de langue partagée par différents locuteurs (mais aussi pays, institutions) qui s’identifient avec le français sous des formes diverses et variées. Ainsi, avoir le français en partage signifie faire partie du monde francophone dans le sens le plus large possible.

${ }^{4}$ Le CARAP est cité en tant que document fédérateur d'un certain nombre de méthodes et d'outils didactiques qui constituent les approches plurielles: la pédagogie intégrée, l'éveil aux langues, l'interculturalité, l'intercompréhension, la didactique du plurilinguisme, etc. Voir : De Pietro \& Gerber [11] pour une synthèse.

${ }_{6}^{5}$ Pour une définition et une description de la variation sociolinguistique du français, voir : Gadet [16].

${ }^{6}$ Voir la loi fédérale sur les langues nationales et la compréhension entre les communautés linguistiques (441.1): https://www.admin.ch/opc/fr/classified-compilation/20062545/index.html.
} 
désignent les différentes L1 territoriales) et les langues étrangères ${ }^{7}$. Cette dernière catégorie s'avère particulièrement problématique, regroupant sous la même étiquette aussi bien les langues nationales (allemand, italien, français et romanche) que des langues étrangères telles que l'anglais ou l'espagnol ${ }^{8}$.

À ce sujet, Dabène ([7] : 35-36) propose - en reprenant la notion de «xénité » élaborée par Weinrich [28] des critères qui permettent de mieux cerner la «distance » entre les langues, à savoir la distance matérielle (éloignement géographique), culturelle et linguistique (typologique). Selon les deux premiers critères, on pourrait difficilement qualifier le français de langue « étrangère » en Suisse, si l'on suppose l'existence d'un minimum de culture commune entre les différentes régions linguistiques, au-delà de différences intrinsèques (voir : Büchi [2]). Quant au critère de distance linguistique, celui-ci nécessiterait sans doute d'être nuancé par rapport à l'allemand - le seul à ne pas relever de la famille des langues romanes - mais ne peut certainement pas s'appliquer à l'italien et au romanche.

Enfin, la catégorie de « langues nationales » n'est pas moins problématique, car il faudrait avant tout s'entendre sur la signification qu'on lui attribue : à savoir un synonyme de langues parlées sur le territoire, avec une claire acception politico-administrative, ou bien des langues «en commun », qui font partie intégrante du paysage socio- et éco-linguistique helvétique, en lien avec les identités collectives (voir : Widmer [29]). La deuxième acception nous paraît mieux correspondre au visage actuel d'un pays de plus en plus urbain et interconnecté.

Pour essayer de répondre à ces questionnements, nous allons décrire la place du français dans le système éducatif tessinois, notamment par le truchement d'une enquête auprès de lycéens à Locarno, de façon à fournir les points de repère nécessaires aux analyses ultérieures et d'ouvrir sur les perspectives futures.

\section{Le système éducatif au Tessin : la place du français}

En Suisse, il n'existe pas de système éducatif unique au niveau fédéral. Ce dernier relève de la compétence des cantons, ce qui fait qu'on peut compter 26 systèmes éducatifs différents. Il s'agit dès lors d'un système très diversifié quant à l'organisation et à la durée des degrés scolaires et, notamment, en ce qui concerne le contenu des plans d'études. Néanmoins, des tentatives d'harmonisation ont vu le jour ces dernières années, qui ont abouti à la mise en place d'un accord intercantonal sur l'harmonisation de la scolarité obligatoire (HarmoS), entré en vigueur en 2009 et qui sera graduellement mis en application jusqu'en 2019. Au Tessin, le système HarmoS s'applique à partir de cette année scolaire 2015/2016. Pour ce qui concerne le degré scolaire post-obligatoire (secondaire II et tertiaire), la formation relève de la compétence d'organismes intercantonaux. Ainsi, par exemple, le canton du Tessin est regroupé avec la Suisse romande, sous la responsabilité de la CIIP (voir supra).

\subsection{L'enseignement des langues nationales et étrangères}

Dans un pays plurilingue tel que la Suisse, l'enseignement des langues revêt une importance particulière. Dès les années 70, la CDIP recommandait l'enseignement d'une deuxième langue nationale à l'école primaire, qui vient s'ajouter à l'étude de la langue de scolarisation territoriale. En 2009 , le modèle $5^{\mathrm{e}} / 7^{\mathrm{e}}$ mis en place dans le cadre du concordat HarmoS prévoit l'apprentissage d'une première langue étrangère à partir de la $5^{\mathrm{e}}$ année de scolarité (élèves de 8-9 ans) et d'une deuxième langue étrangère au cours de la $7^{\mathrm{e}}$ année (élèves de 10-11 ans). Les langues enseignées à ce stade sont une deuxième langue nationale et l'anglais. L'ordre d'introduction dépend des cantons. Le Tessin connaît une situation particulière par rapport aux autres cantons, car il propose l'étude de trois langues pendant la scolarité obligatoire, deux langues nationales (le français dès la $5^{\mathrm{e}}$, l'allemand dès la $9^{\mathrm{e}}$ ) et $1^{\prime}$ 'anglais (dès la $10^{\mathrm{e}}$ ). L'introduction de l'anglais en $10^{\mathrm{e}}$ année de scolarité (élèves de 13-14 ans), instaurée en 2004, a eu pour conséquence un changement de statut du français. Celui-ci, en effet, était obligatoire jusqu'à la fin du secondaire I $\left(10^{\mathrm{e}}-11^{\mathrm{e}}\right.$ années $)$, depuis il est devenu optionnel.

En ce qui concerne l'enseignement post-obligatoire, deux voies sont possibles en Suisse : une voie de formation générale (les écoles de maturité gymnasiale et les écoles de culture générale), qui prépare aux filières du degré tertiaire, et une voie de formation professionnelle, qui qualifie pour la pratique d'une profession. En 2013, la CDIP a approuvé une stratégie pour l'enseignement des langues pour le degré secondaire II, qui n'a pas encore été adoptée à 1'échelle nationale ${ }^{10}$. Au Tessin, l'enseignement post-obligatoire dure normalement quatre ans. Le lycée de Locarno, dans lequel s'est déroulée notre enquête, est l'un des 6 établissements du secondaire II du canton, qui comporte cinq lycées cantonaux (Bellinzone, Lugano 1, Lugano 2, Locarno et Mendrisio) et une École de commerce (Bellinzone). L'enseignement des langues se distribue sur les quatre années d'études, avec plusieurs configurations possibles. Il prévoit l'étude obligatoire d'une deuxième langue nationale (français ou allemand) et d'une autre langue au choix entre le français, l'allemand, l'anglais, l'espagnol, le latin et le grec. Le français, tout comme l'allemand, peut être choisi comme "option spécifique », c'est-à-dire avec une à deux heures G'approfondissement hebdomadaires

Voir, par exemple, le rapport de la Commission de la science, de l'éducation et de la culture du Conseil national du 15 septembre 2006 à propos de l'Initiative parlementaire « Loi fédérale sur les langues nationales et la compréhension entre les communautés linguistiques », en particulier le glossaire à la fin du document.

${ }^{8}$ Plusieurs auteurs ont déjà relevé le caractère imprécis et problématique de ces catégories, notamment lorsqu'on essaie de les appliquer à des contextes précis. Voir : Dabène ([7]: 7-38), pour une synthèse.

${ }^{9}$ Weinrich [28] définit la «xénité » comme l'interprétation de l'altérité d'une langue, à savoir la perception d'un ensemble de traits permettant de mesurer la distance entre les langues.

${ }^{10}$ Source : http://www.edudoc.ch/static/web/dokumentation/sprachenstrat sek2 f.pdf. 
supplémentaires. Depuis 1995, des filières de formation bilingues ont été créées en Suisse. Elles offrent la possibilité de suivre des cours « en immersion », c'est-à-dire l'enseignement de disciplines non linguistiques dans l'une des langues nationales (voir: Gajo [13]). Au Tessin, une filière bilingue italien-français n'existe actuellement qu'à l'École de commerce de Bellinzone. Depuis 2014/2015, un cursus bilingue italien-allemand est également possible au lycée de Locarno. À titre d'illustration, en 2006, 40\% des établissements scolaires du degré secondaire II proposaient des formations bilingues (Elmiger [12]).

\subsection{La place du français}

Grâce aux données qui nous ont été fournies par la direction du lycée cantonal de Locarno, nous sommes en mesure de reconstituer les choix linguistiques des élèves qui entrent en première année de lycée sur les quinze dernières années (2000-2015). Les chiffres montrent une baisse importante du nombre d'élèves qui choisissent de continuer ou de reprendre l'étude du français au secondaire II (Fig. 1) et livrent ainsi un large aperçu de la place qu'occupe la langue française auprès d'un segment de population crucial : la jeunesse.

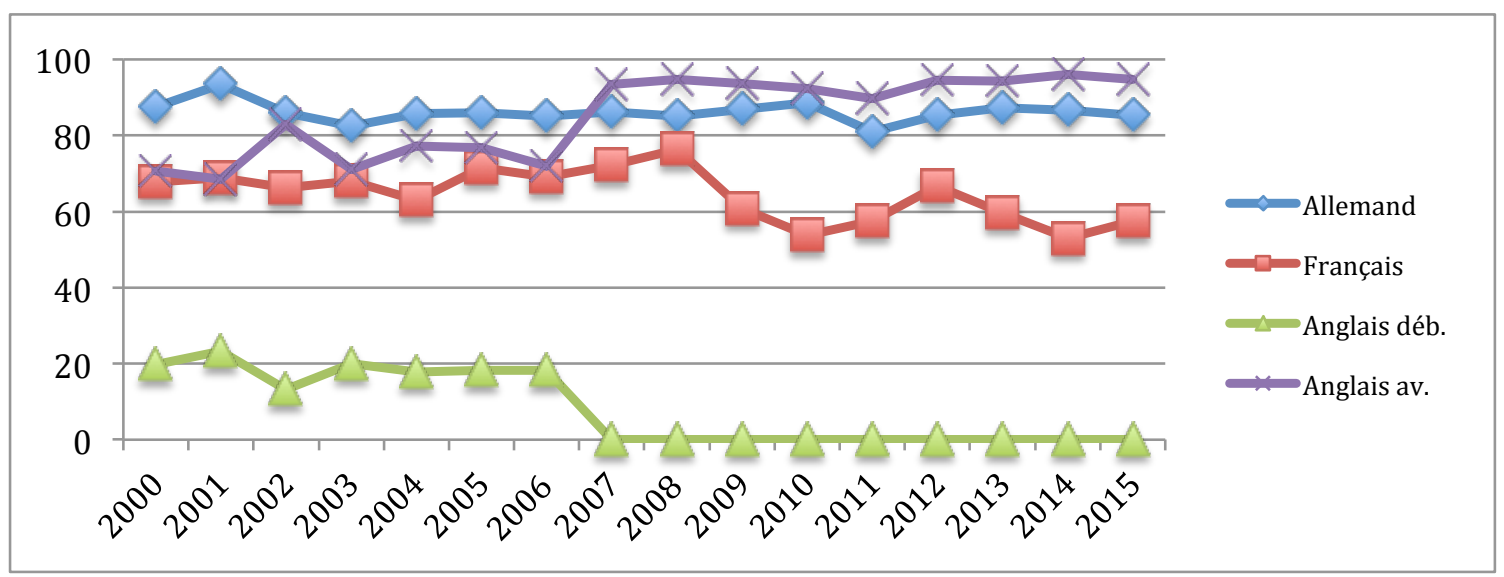

Fig. 1. Choix linguistiques des lycées de Locarno de 2000 à 2015.

Source : Direction du lycée cantonal de Locarno

Le pourcentage d'élèves francisants en première année, en effet, s'élevait à environ $70 \%$ entre 2000 et 2008 . Puis, il est descendu autour de 58\% en moyenne, entre 2009 et 2015, ce qui représente une baisse d'environ $12 \%$.

Parallèlement, il peut être constaté (Fig. 1) une hausse dans le nombre d'élèves qui choisissent l'anglais (niveau avancé). Leur pourcentage est passé de $74 \%$ en moyenne entre 2000 et 2006 à environ $83 \%$ entre 2007 et 2015 , soit une augmentation de 9\%. L'anglais (niveau débutant), qui était choisi par 18\% des élèves jusqu'en 2006, n'est plus proposé à partir de 2007, année dans laquelle s'inscrivent au lycée des élèves ayant déjà fait de l'anglais pendant deux ans au secondaire I. C'est en 2004, en effet, qu'entre en vigueur au Tessin la «Riforma 3 »" , qui prévoit l'introduction de l'anglais en $10^{\mathrm{e}}$ et $11^{\mathrm{e}}$ années (voir supra). Le français, qui était obligatoire jusque là pendant les quatre années d'école moyenne, est désormais facultatif les deux dernières années. Les années 2007-2009 constituent donc des années charnière. Faut-il y voir un effet des politiques linguistiques sur le choix des élèves?

La comparaison du nombre d'élèves francisants du lycée de Locarno avec le pourcentage d'élèves francisants des autres établissements tessinois en 2014/2015, fourni par le Département de l'Instruction publique du Tessin, nous permet de relever un taux comparable pour l'ensemble du canton (Tab. 1).

\begin{tabular}{lccc}
\multicolumn{1}{c}{ Lycée } & Elèves francisants & Élèves Lycée & $\mathbf{\%}$ \\
\hline Bellinzone & 348 & 717 & $48,50 \%$ \\
Locarno & 302 & 643 & $50 \%$ \\
Lugano 1 & 580 & 1089 & $53 \%$ \\
Lugano 2 & 290 & 683 & $42,50 \%$ \\
Mendrisio & 296 & 619 & $48 \%$
\end{tabular}

Tab. 1. Pourcentage de lycéens francisants au Tessin en 2014/2015, tous degrés confondus. Source : Département de l'instruction publique, de la culture et du sport du canton du Tessin

\footnotetext{
${ }^{11}$ http://www4.ti.ch/decs/ds/cerdd/scuoladecs/riforma3/nome/.
} 
En ce qui concerne le choix de l'option spécifique au lycée de Locarno, 6\% des élèves en moyenne ont choisi le français entre 2000 et 2007. De 2008 à 2015, ce pourcentage baisse à $0 \%$, avec une exception en 2009, où $5 \%$ des élèves l'ont choisi (Fig. 2).

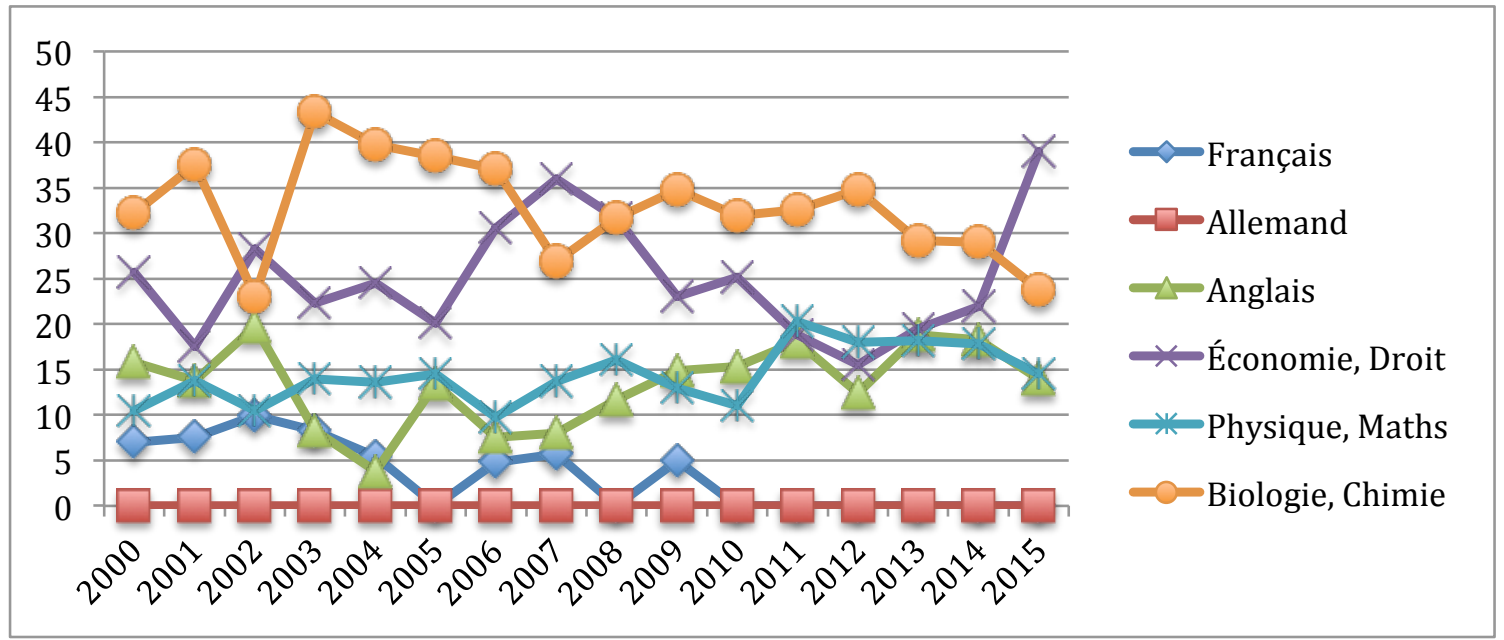

Fig. 2. Choix de l'option spécifique au lycée de Locarno de 2000 à 2015. Source : Direction du lycée cantonal de Locarno

L'anglais enregistre une légère hausse. $11 \%$ des élèves l'ont choisi entre 2000 et 2007 . Ils sont $15 \%$ entre 2008 et 2015. Cependant, si l'on regarde ces chiffres en tenant compte de la situation globale des autres disciplines, ce sont surtout l'économie, le droit et les matières scientifiques (en particulier la biologie et la chimie) qui semblent l'emporter sur les langues.

\section{Une enquête auprès de lycéens à Locarno}

Ces données chiffrées et officielles à propos des choix linguistiques des élèves fournies par les instances publiques tessinoises méritent d'être enrichies par l'analyse des attitudes et des représentations des mêmes élèves vis-à-vis de la langue française et de ses variations. Les réponses à ces questions fourniront des éléments complémentaires à la réflexion socio-didactique sur la place et le statut de l'enseignement du français en Suisse.

\subsection{Description du cadre général de l'enquête}

L'enquête présentée ici se fonde sur un questionnaire papier ${ }^{12}$ qui a été administré grâce à la collaboration des enseignants à un échantillon de 214 élèves du lycée cantonal de Locarno, soit la quasi-totalité des élèves francisants de l'établissement. Cet échantillon comporte 76 jeunes hommes et 138 jeunes filles, âgés de 16,5 ans en moyenne, et qui ont derrière eux environ 9 années d'études du français. $10 \%$ déclarent être de nationalité étrangère et $32 \%$ se disent plurilingues. Parmi les langues parlées figurent, par ordre alphabétique : l'allemand, l'anglais, l'arabe, le catalan, le dialecte tessinois, l'espagnol, le français, l'italien, le japonais, le macédonien, le polonais, le portugais, le serbo-croate et le suisse allemand.

La passation s'est faite de manière individuelle, en classe, et a duré une quinzaine de minutes. Les principes qui ont présidé aux choix des questions étaient de nature sociodidactique et cherchaient à dresser un portrait de l'image que les jeunes Tessinois se font du français parlé en Suisse ${ }^{13}$. Dès lors, les questions proposées ne mettent jamais la langue française en contraste avec l'anglais ni avec d'autres langues. Notre objectif, en effet, n'est pas d'évaluer l'attractivité du français en opposition à d'autres langues, mais de mieux cerner la place et le statut de la langue française en Suisse aujourd'hui.

\footnotetext{
${ }^{12}$ Le choix d'administrer un questionnaire papier est lié au nombre important d'élèves interrogés. Pour le volet « enseignants » de ce projet, nous privilégierons l'enregistrement d'entretiens.

${ }^{13}$ Le français parlé en Suisse romande se caractérise par un certain nombre de traits variationnels qui le distinguent du français hexagonal. L'intercompréhension avec les autres variétés de français n'est jamais compromise, hormis quelques éléments du lexique (régionalismes, archaïsmes, emprunts aux autres langues nationales, créations endogènes) ou et - surtout - quelques spécificités phonétiques. Notre objectif n'est pas de fournir une description détaillée de ces traits. Pour une présentation plus exhaustive, nous renvoyons à Andreassen et al. [1].
} 


\subsection{Présentation du questionnaire}

\subsubsection{Généralités}

Le questionnaire comporte six questions à choix multiple ${ }^{14}$. Les quatre premières visent, d'une part, à examiner les attitudes et les représentations des élèves vis-à-vis du français. D'autre part, elles cherchent à savoir si les élèves sont sensibles à la thématique de la variation et, plus précisément, quelle est la variété de français étudiée à l'école ou quelle est la forme de français qu'ils aimeraient parler. Les questions 5 et 6 , enfin, sont davantage liées au questionnaire sociodémographique et visent donc à obtenir des informations quant à l'avenir proche des élèves. Les questions 1,5 et 6 proposent 4 réponses, les questions 2 et 3 en proposent 5 , mais avec une seule réponse possible. La question 4 propose 12 réponses différentes, avec choix du nombre de réponses libre.

\subsubsection{Détails sur le contenu et la portée des questions}

La première question porte sur le statut du français en Suisse et cherche à savoir si celui-ci est perçu par les élèves comme une langue étrangère ordinaire ou comme une langue «nationale », importante pour vivre, étudier et travailler en Suisse. Considérer le français comme une langue étrangère ordinaire, en effet, reviendrait à dire que celui-ci ne revêt pas un statut particulier aux yeux des élèves et qu'il serait donc à placer au même niveau que des langues telles que l'anglais ou l'espagnol. En revanche, tenir le français pour une langue nationale du pays ou, dans une moindre mesure, une langue importante pour vivre, étudier et travailler en Suisse, assignerait un rôle spécifique à la langue française dans le paysage sociolinguistique helvétique.
Question 1 : qu'est-ce que le français pour toi ?
A C'est une langue étrangère qu'on étudie à l'école
B C'est une des langues nationales de mon pays
C C'est une langue importante pour vivre, étudier et travailler en Suisse
D Je ne sais pas

La deuxième question interroge la sensibilité des élèves envers la variation et notamment la variété de français étudiée à l'école. L'objectif de cette question est de savoir si les élèves associent le français qu'ils étudient à la langue d'un pays étranger (la France) ou à la langue de leur pays (la Suisse). Les réponses « français standard» et «français standard, avec quelques spécificités de Suisse romande » font figure de réponses «moyennes », pour le cas où les élèves n'associeraient pas la langue française à un pays et préféreraient rester neutres.

\section{Question 2 : quel français tu étudies à l'école ? \\ A Le français de France \\ B Le français standard \\ C Le français de Suisse romande \\ D Le standard, avec quelques spécificités régionales \\ E Je ne sais pas}

La question trois s'intéresse à la façon dont les élèves aimeraient s'exprimer en français (comme un Français, comme un Suisse romand, de façon neutre, etc.) et suit la même logique que la question 2. En fonction de la réponse, les élèves indiqueront qu'ils s'identifient davantage avec un Français ou avec un Suisse romand. Ou alors, ils pourront opter pour une réponse moyenne en affirmant qu'ils souhaitaient s'exprimer de façon neutre, ou de façon neutre avec quelques spécificités de Suisse romande.
Question 3 : comment tu aimerais t'exprimer en français ?
A Comme un Français
B Comme un Suisse romand
C De façon neutre
D De façon neutre, avec quelques spécificités régionales
E Je ne sais pas

La quatrième question, à visée plus concrète, cherche à savoir si les élèves estiment que le français est une langue importante pour la Suisse et dans quels domaines : la formation et la vie professionnelle (étudier et/ou travailler), les loisirs et la vie culturelle (voyager, lire, écouter de la musique, faire la fête et s'amuser, surfer sur internet), la vie en Suisse (interagir avec d'autres Suisses, regarder la télé et s'informer, rester en contact avec les amis, interagir à l'armée,

\footnotetext{
${ }^{14}$ Le questionnaire peut paraître succinct à première vue. Cependant, le nombre limité de questions relève d'un choix délibéré. La difficulté de retenir l'attention de jeunes adolescents est, en effet, connue de tous. De plus, ce questionnaire ne représente qu'une partie de l'enquête menée au lycée cantonal de Locarno et vise à compléter les entretiens qualitatifs menés auprès des enseignants et des membres de la direction (voir : Paternostro, [22]).
} 
suivre la politique fédérale). Selon les domaines, l'importance du français se profilera de manière différente, davantage orientée vers des questions pragmatiques (avenir personnel et professionnel), centrée sur les loisirs et la vie culturelle, ou en lien avec des thématiques identitaires (la vie en Suisse).
Question 4 : selon ton expérience, le français est important pour
A Étudier ou travailler
G Suivre la politique fédérale
B Surfer sur internet
$\mathbf{H}$ Rester en contact avec les amis
C Regarder la télé, s'informer
I Interagir à l'armée
D Faire la fête, s'amuser
J Interagir avec d'autres suisses
E Lire, écouter de la musique
K Autre
F Voyager
L Ce n'est pas important

Enfin, les questions cinq et six, qui sont fermées (oui, non, peut-être, ne sais pas), visent à savoir si les élèves envisagent de faire des études en Suisse romande (question cinq) et s'il est intéressant pour eux de mieux connaître le français de Suisse romande (question six).

\subsection{Présentation des résultats}

Le traitement des résultats s'est déroulé de la façon suivante : les questionnaires ont été dépouillés individuellement, les résultats pour chaque réponse ont été additionnés. Puis, un calcul de pourcentage a été effectué pour chaque item. Enfin, le test statistique chi-carré a été appliqué aux résultats de chaque question, de manière à exclure l'hypothèse nulle, à savoir que les réponses données ne sont pas dues au hasard. Tous les résultats sont statistiquement significatifs.

\subsubsection{Première question}

$48 \%$ des élèves tessinois considèrent le français comme une langue importante pour vivre, étudier et travailler en Suisse et $44 \%$ d'entre eux perçoivent le français comme une des langues nationales du pays. Seulement $8 \%$ le considèrent comme une langue étrangère ordinaire (Tab. 2).

\begin{tabular}{|l|c|}
\hline Question 1 : qu'est-ce que le français pour toi ? & $\%$ \\
\hline A C'est une langue étrangère qu'on étudie à l'école & 8 \\
\hline B C'est une des langues nationales de mon pays & 44 \\
\hline C C'est une langue importante pour vivre, étudier et travailler en Suisse & 48 \\
\hline D Je ne sais pas & 0 \\
\hline
\end{tabular}

Tab. 2. Réponse à la question 1 .

\subsubsection{Deuxième question}

$44 \%$ des élèves considèrent le français qu'ils étudient à l'école comme le français standard. Pour 39,5\% d'entre eux la variété étudiée au lycée correspond au français de France. Seuls 5\% croient étudier le français standard avec quelques spécificités régionales et $0,5 \%$ le français de Suisse. Cependant, $11 \%$ affirment ne pas avoir d'avis, ce qui représente une proportion non négligeable (Tab. 3).

Tab. 3. Réponses à la question 2 .

\begin{tabular}{|l|c|}
\hline Question 2 : quel français tu étudies à l'école ? & $\%$ \\
\hline A Le français de France & $\mathbf{3 9 , 5}$ \\
\hline B Le français standard & $\mathbf{4 4}$ \\
\hline C Le français de Suisse romande & $\mathbf{0 , 5}$ \\
\hline D Le standard, avec quelques spécificités régionales & $\mathbf{5}$ \\
\hline E Je ne sais pas & $\mathbf{1 1}$ \\
\hline
\end{tabular}

\subsubsection{Troisième question}

62,5\% des élèves souhaiteraient s'exprimer comme un Français. 21\% affirment vouloir s'exprimer de façon neutre. 7\% préfèreraient s'exprimer de façon neutre, mais avec quelques spécificités régionales. Seuls 6,5\% aimeraient parler comme un Suisse romand (Tab. 4)

\begin{tabular}{|l|c|}
\hline Question 3 : comment tu aimerais t'exprimer en français ? & $\mathbf{\%}$ \\
\hline A Comme un Français & $\mathbf{6 2 , 5}$ \\
\hline B Comme un Suisse romand & $\mathbf{6 , 5}$ \\
\hline C De façon neutre & $\mathbf{2 1}$ \\
\hline D De façon neutre, avec quelques spécificités régionales & $\mathbf{5}$ \\
\hline E Je ne sais pas & $\mathbf{3}$ \\
\hline
\end{tabular}

Tab. 4. Réponses à la question 3 . 


\subsubsection{Quatrième question}

Les réponses à la question quatre ont mis en évidence que les activités pour lesquelles les jeunes tessinois considèrent le français comme important sont : voyager (94\%), étudier et/ou travailler (92,5\%), interagir avec d'autres Suisses (70\%), lire et écouter de la musique (41\%), regarder la télévision et s'informer $(28,5 \%)$, rester en contact avec les amis $(27 \%)$, surfer sur internet (12\%), faire la fête et s'amuser (12\%), interagir à l'armée (10\%), suivre la politique fédérale (11\%). Seul $1 \%$ considèrent que le français n'est pas important (Tab. 5).

Tab. 5. Réponses à la question 4 .

\begin{tabular}{|l|c|}
\hline Question 4 : selon ton expérience, le français est important pour & $\mathbf{\%}$ \\
\hline A Étudier ou travailler & $\mathbf{9 2 , 5}$ \\
\hline B Surfer sur internet & $\mathbf{1 2}$ \\
\hline C Regarder la télé, s'informer & $\mathbf{2 8 , 5}$ \\
\hline D Faire la fête, s'amuser & $\mathbf{1 2}$ \\
\hline E Lire, écouter de la musique & $\mathbf{4 1}$ \\
\hline F Voyager & $\mathbf{9 4}$ \\
\hline G Suivre la politique fédérale & $\mathbf{1 1}$ \\
\hline H Rester en contact avec les amis & $\mathbf{2 7}$ \\
\hline I Interagir à l'armée & $\mathbf{1 0}$ \\
\hline J Interagir avec d'autres suisses & $\mathbf{7 0}$ \\
\hline K Autre & $\mathbf{2}$ \\
\hline L Ce n'est pas important & $\mathbf{1}$ \\
\hline
\end{tabular}

\subsubsection{Cinquième et sixième questions}

En ce qui concerne la question cinq («Tu penses faire des études en Suisse romande ?»), c'est le «peut-être » qui l'emporte, avec $49 \%$ des réponses, suivi de « oui » $(31 \%)$, «non» $(11 \%)$ et « je ne sais pas $(9 \%)$. À la question 6 (« Tu penses que ce serait important de mieux connaître le français de Suisse romande ?»), 39\% des élèves ont répondu « oui », $35 \%$ « peut-être, $17 \%$ « non » et $9 \%$ « je ne sais pas ».

\subsection{Synthèse et discussion des résultats}

L'objectif de cette enquête était à la fois d'examiner les attitudes et les représentations des élèves tessinois vis-à-vis du français et de savoir si ces derniers sont sensibles à la variation sociolinguistique, notamment à la variété de français étudiée à l'école et à la forme qu'ils aimeraient parler. Les réponses sont censées constituer des éléments-clé pour l'analyse socio-didactique plus globale de la place et du statut de l'enseignement du français dans des contextes plurilingues et multiculturels tels que la Suisse.

Les résultats de cette enquête semblent souligner la place particulière que la langue française occupe en Suisse. Il apparait en effet que pour les élèves tessinois ( $48 \%$ ) le français est une langue importante pour le pays (question un). En outre, si on additionne le pourcentage de ceux qui le considèrent comme l'une des langues nationales (44\%), on atteint un score de $92 \%$. Le français n'est donc clairement pas une langue étrangère ordinaire (seuls $8 \%$ des élèves interrogés pensent ainsi), mais une langue «nationale », importante pour vivre, étudier et travailler en Suisse.

Les résultats de cette enquête suggèrent aussi que les élèves associent le français qu'ils étudient à l'école non pas à la norme locale (le français de Suisse), mais au français standard (44\%) et au français de France (39,5\%). Ces deux pourcentages cumulés représentent $83,5 \%$ des réponses. Ce qui semble contraster avec le statut privilégié que les élèves eux-mêmes attribuent au français en Suisse (réponses à la question un). Ces données méritent néanmoins d'être interprétées avec prudence, car on peut se demander ce que les élèves savent réellement des différences entre français standard, français de France, français de Suisse (voir note 14). On se souvient, en effet, que $11 \%$ des élèves semblent ne pas être en mesure d'identifier la variété étudiée. Ce qui pourrait être le signe d'une absence de réflexion sur la variation à l'école.

Concernant la façon dont les élèves tessinois aimeraient s'exprimer (question trois), en lien avec ce qui précède, il n'est pas surprenant de constater que la majorité souhaiterait parler comme un Français et non comme un Suisse romand (62,5\% vs. 6,5\%). Ce résultat pourrait s'inscrire dans la même tendance relevée par des études antérieures chez les locuteurs francophones de régions périphériques telles que la Suisse. Ces derniers, en effet, entretiennent une relation ambivalente avec leur langage, qui alterne valorisation et dépréciation et qui est révélatrice d'un sentiment d'insécurité linguistique (Singy [27] ; Moreau et al. [19] ; Prikhodkine [23] ; Racine et al. [24]). Encore une fois, il faudrait chercher à savoir à quoi renvoient chez les élèves des notions telles que "parler comme un Français » ou "parler comme un Suisse romand ».

Les réponses à la question quatre donnent une indication quant aux domaines pour lesquels le français joue un rôle important. Les items ayant obtenu le score le plus élevé concernent la mobilité, la formation, la vie professionnelle et le contact avec les autres Suisses. Parmi les items restants, le français joue un rôle dans la vie culturelle (lire et écouter de la musique) et il constitue aussi un moyen d'accès aux médias et à l'information (s'informer, surfer sur Internet, suivre 
l'actualité politique). Le score presque nul (1\%) qu'a obtenu l'item « ce n'est pas important» semble attester de l'attachement et de la reconnaissance des élèves tessinois envers le français.

Enfin, les réponses aux questions cinq et six montrent qu'une partie non négligeable des élèves pensent poursuivre leurs études en Suisse romande et surtout qu'ils seraient intéressés à mieux connaître le français régional.

Ces résultats nous ont ainsi livré un aperçu des attitudes et des représentations des élèves tessinois vis-à-vis de la langue française. Il en ressort une vision pragmatique, clairement en lien avec les besoins et les pratiques socioprofessionnels. Cependant, ils ont aussi permis de relever que le rôle central que les élèves attribuent au français en Suisse contraste avec la variété étudiée à l'école, qu'ils identifient avec le français standard et/ou de France, et l'intérêt manifesté envers la façon de parler des Français.

Du point de vue des élèves, intégrer une réflexion sur la variation (notamment régionale) serait donc une façon de répondre à leurs besoins tout en les préparant au contact avec la réalité de la langue à laquelle ils seront confrontés sur le terrain et en les aidant à développer des attitudes plus positives envers la norme locale, en accord avec la place particulière que la langue française occupe dans le pays à leurs yeux. Cette réflexion pourrait prendre appui sur la situation sociolinguistique du Tessin et se nourrir ainsi de l'expérience que les élèves ont de l'italien langue « nationale » et de ses traits régionaux par rapport au standard d'Italie. L'objectif de cette démarche, qui émane d'une approche didactique intégrée et contextualisée (voir plus haut), serait ainsi de développer chez les élèves « un sentiment de lien, une certaine familiarité, éventuellement un sentiment de participation ou d'implication, qui concerne la languecible et les contextes politiques, sociaux et culturels dans lesquels elle s'utilise » (Grin [15] : 33). Les avantages seraient multiples : aider les élèves à faire le rapprochement entre le français qu'ils considèrent comme une langue importante en Suisse et la langue qu'ils apprennent en cours ; stimuler leur intérêt envers une langue qu'ils ont déjà en partage ; les encourager à poursuivre l'apprentissage du français jusqu'à la fin de leur scolarité ; promouvoir le plurilinguisme et la diversité en tant qu'éléments-clé du paysage linguistique et culturel de la Suisse.

\section{Conclusion et perspectives}

Cet article a réfléchi à l'intérêt que représentent des approches didactiques intégrées et contextualisées pour l'enseignement du français dans des contextes "complexes» tels que la Suisse plurilingue et multiculturelle. Le paysage sociolinguistique helvétique, et tout particulièrement le canton italophone du Tessin, s'est révélé un terrain fécond pour l'observation et l'analyse des aspects socio-institutionnels et des attitudes des élèves vis-à-vis de la langue française et de ses variations, contribuant ainsi à mieux définir la place que le français occupe dans le contexte sociodidactique de la Suisse.

Si d'un point de vue institutionnel, le français semble en perte de vitesse par rapport à d'autres langues ou à d'autres disciplines, l'analyse des représentations des élèves tessinois et de leur sensibilité à la variation a permis de montrer que le terrain helvétique est encore propice à la diversité linguistique et culturelle et que le français n'a pas fini de jouer un rôle dans le pays.

Une vision intégrée et contextualisée des français de Suisse et les implications didactiques qui en découlent ont fait émerger deux orientations principales qui méritent d'être explorées : la découverte de la variabilité et de la diversité linguistiques et la prise de conscience du français en tant que langue «nationale ». Elles constituent deux moyens privilégiés d'amener les élèves-apprenants à se reconnaître en tant que sujets sociaux étant eux-mêmes partie prenante de la « francophonie Suisse ». Différents outils pédagogiques pourraient être élaborés en ce sens, d'autres existent déjà et pourraient être davantage exploités pour favoriser l'accès à une connaissance plus approfondie des composantes linguistiques et culturelles et permettant d'employer le français appris en classe dans la vie réelle : l'accès aux médias, à la littérature et, plus généralement, au monde socio-culturel romand ; les échanges linguistiques, les filières bilingues et l'investissement dans des projets communs avec les autres francophones de Suisse, etc.

Cette étude ne représente qu'une étape de l'analyse socio-didactique du terrain helvétique. Elle met en évidence la nécessité de poursuivre notre réflexion en nous intéressant aussi aux représentations et aux pratiques des acteurs de l'enseignement. Les éléments issus de cette première enquête seront ainsi complétés par le volet « enseignants ». Grâce à des entretiens menés auprès des enseignants de français et des responsables administratifs du lycée cantonal de Locarno, on comparera les réponses des élèves avec ce qui se passe sur le terrain. Il s'agira également de comprendre, en amont, dans quelle mesure les enseignants ont été sensibilisées ou sont sensibles à la thématique de la variation et du statut particulier que le français revêt en Suisse. En aval seront abordées des problématiques telles que les normes et les pratiques pédagogiques, la définition d'objectifs contextualisés, la mise au point de dispositifs adaptés.

\section{Bibliographie}

[1] H., Andreassen, R., Maître, I., Racine, La Suisse. In : Detey, S., Durand, J., Laks, B. \& Lyche, C. (dir.) Les variétés du français parlé dans l'espace francophone. Ressources pour l'enseignement (Ophrys, Paris, 213-227, 2010)

[2] C., Büchi, Mariage de raison. Romands et Alémaniques : une histoire suisse (Zoé, Genève, 2015)

[3] L., Cadet, E., Guerin, (dir.) FLM, FLS, FLE au delà des catégories. Le français aujourd'hui (A. Colin, Paris, 2012)

[4] M. Candelier et al., CARAP. Cadre de référence pour les Approches Plurielles des Langues et des Cultures (En ligne : http://carap.ecml.at, 2007) 
[5] H., Christen, 'Tour de Suisse' der Deutschschweizer Dialekte. In : Forum Helveticum (éd.), Dialekt in der (Deutsch)Schweiz - Zwischen lokaler Identität und nationaler Kohäsion. Le dialecte en Suisse (alémanique) - entre identité locale et cohésion nationale, Forum Helveticum, Schriftenreihe 15, 21-25 (2005)

[6] C., Cordier-Gauthier, C., Dion, Français langue seconde, université et recherche au Canada, en Belgique, en Suisse et en France. In: M., Verdelhan-Bourgade (dir.) Le français langue seconde: un concept et des pratiques en évolution (De Boeck, Bruxelles, 29-83, 2007)

[7] L., Dabène, Repères sociolinguistiques pour l'enseignement des langues (Hachette, Paris, 1994)

[8] L., Dabène, M., Rispail, La sociodidactique : naissance et développement d'un courant au sein de la didactique du français en France. Lettre de l'AIRDF 1, 10-13 (2008)

[9] J.-F., De Pietro, L'école face aux variétés du français : réflexions à partir de la situation en Suisse francophone. $L a$ langue française dans sa diversité (Ministère de la Culture, des Communications et de la Condition féminine, Québec, 181-208, 2008)

[10] J.-F., De Pietro, M., Rispail (dir.), L'enseignement du français à l'heure du plurilinguisme (Presses universitaires de Namur, Namur, 2014)

[11] J.-F., De Pietro, B., Gerber, Les approches plurielles des langues et des cultures. Babylonia 2, (2015)

[12] D., Elmiger, La maturité bilingue en Suisse. La mise en œuvre variée d'une innovation de la politique éducative (Secrétariat d'État à l'éducation et à la recherche, Berne, 2008)

[13] L., Gajo, Politiques éducatives et enjeux socio-didactiques : l'enseignement bilingue francophone et ses modèles. Glottopol 13, 14-27 (2009)

[14] F., Grin, L’aménagement linguistique en Suisse. Télescope 16, 3, 55-74 (2010)

[15] F., Grin, Le débat sur les langues en quinze questions : arguments, faits et chiffres (Observatoire Économie Langues - Formation, Université de Genève, Faculté de traduction et d'interprétation, Genève, 2014)

[16] F., Gadet, La variation sociale en français, 2 édition (Ophrys, Paris, 2007)

[17] E. Guerin, Le français, "langue maternelle" est-il une langue "vivante" ? Réflexion sur la place de la variation stylistique dans le discours scolaire. LIDIL 50, 147-178 (2014)

[18] G., Lüdi, I., Werlen, Le Paysage linguistique en Suisse. Recensement fédéral de la population 2000 (Office fédéral de la statistique, Neuchâtel, 2005)

[19] M.-L., Moreau, P., Bouchard, S., Demartin, F., Gadet, E., Guerin, B., Harmegnies, K., Huet, F., Laroussi, A., Prikhodine, P., Singy, N., Thiam, H., Tyne, Les accents de la francophonie. Une enquête internationale. Français \& Société 16 (2007)

[20] E., M., Pandolfi, L'italiano nostro e degli altri. Le varietà dell'italiano. I Quaderni dell'Associazione Carlo Cattaneo 67 (2011)

[21] R., Paternostro, La transcription de données orales en classe de français : un outil d'éveil à la variation en FLE et en FLM ?. In : Cadet, L. \& Pégaz, A. (éd.) Les langues à l'école, la langue de l'école : quelles configurations didactiques? quelles reconfigurations de la formation? (Presses Universitaires de l'Artois, Arras, sous presse)

[22] R., Paternostro, Perspectives socio-didactiques dans l'enseignement du FLS / FLE en Suisse : enquête préliminaire au Tessin. Actes du colloque VALS-ASLA 2016, (sous presse)

[23] A. Prikhodkine, Dynamique normative du français en usage en Suisse romande : enquête sociolinguistique dans les cantons de Vaud, Genève et Fribourg (L'Harmattan, Paris, 2011)

[24] I., Racine, S., Schwab, S., Detey, Accent(s) suisse(s) ou standard(s) suisse(s) ? Approche perceptive dans quatre régions de Suisse romande. In : A. Falkert (dir.) La perception des accents du français hors de France (Éditions CIPA, Mons, 41-59, 2013)

[25] E., Roulet, Langue maternelle et langues secondes - vers une pédagogie intégrée (Hatier, Paris, 1980)

[26] P., Singy, L'image du français en Suisse romande. Une enquête sociolinguistique en Pays de Vaud (L'Harmattan, Paris, 1996)

[27] A., Thibaut, Dictionnaire suisse romand (Zoé, Genève, 1997)

[28] H., Weinrich, Petite xénologie des langues étrangères. Communications 43, 187-203 (1986)

[29] J., Widmer, Langues nationales et identités collectives. L'exemple de la Suisse (L'Harmattan, Paris, 2004)

\section{Remerciements}

Nous souhaitons exprimer notre gratitude envers Enrica Galazzi et Candido Matasci, sans qui cette enquête n'aurait jamais vu le jour. Notre gratitude va aussi au directeur du lycée cantonal de Locarno, Fulvio Cavallini, à la vicedirectrice, Margherita Noseda, ainsi qu'aux enseignant-e-s et aux élèves pour leur générosité et leur précieuse collaboration. Un grand merci aussi à Isabelle Racine, pour son soutien matériel et scientifique. 